\title{
Discovery of protein modifications using differential tandem mass spectrometry proteomics
}

Paolo Cifani ${ }^{1+}$, Zhi Li ${ }^{2,3+}$, Danmeng Luo ${ }^{1+}$, Mark Grivainis ${ }^{2}$, Andrew M. Intlekofer ${ }^{4}$, David Fenyö $^{* 2,3}$, Alex Kentsis ${ }^{* 1,5}$

1 Molecular Pharmacology Program, Sloan Kettering Institute, Memorial Sloan Kettering Cancer Center, New York, NY, 10021, USA.

2 Institute for Systems Genetics, NYU Grossman School of Medicine, New York, NY, 10016, USA.

3 Department of Biochemistry and Molecular Pharmacology, NYU Grossman School of Medicine, New York, NY, 10016, USA.

4 Human Oncology \& Pathogenesis Program and Department of Medicine, Memorial Sloan Kettering Cancer Center, New York, NY, 10021, USA.

5 Department of Pediatrics, Memorial Sloan Kettering Cancer Center, and Departments of Pediatrics, Pharmacology, and Physiology \& Biophysics, Weill Medical College of Cornell University, New York, NY, 10021, USA. 


\section{TABLE OF CONTENTS}

The following supporting information is available free of charge at ACS website http://pubs.acs.org

Figure S1: SAMPEI parameterization using specific differentially alkylated proteomes.

Figure S2: SAMPEI achieves sensitive and specific unbiased discovery of peptide modifications in complex cellular proteomes.

Figure S3: Absolute sensitivity of SAMPEI, ByOnic, MSFragger, and MaxQuant.

Figure S4: Computational efficiency of SAMPEI.

Figure S5: Distribution of putative modified sites for agnostically identified PTMs.

Figure S6: Representative mass spectra pairs for peptides with either unmodified experimentally induced +57.02 Da modification or biologic +130.02 Da modification of peptide cysteines.

Figure S7: Representative mass spectra pairs for peptides with either unmodified experimentally induced +57.02 Da modification or biologic +146.02 Da modification of peptide cysteines.

Figure S8: Spectral quality of chemically modified peptides detected as multiple or single modification isoforms.

Figure S9: Quantification of itaconate production by LPS-stimulated macrophages. 
Figure S10: IRG1 immunoblot - entire membrane.

Figure S11: GO term enrichment of proteins with +130.02 Da and +146.02 Da peptide modifications.

Figure S12: Schematic of itaconate reactions with purified BSA and KEAP1 proteins, followed by proteolysis and peptide chromatography and high-resolution tandem mass spectrometry.

Figure S13: Agnostic PTM profiling of itaconate-reacted purified BSA and KEAP1 in vitro, as studied by tryptic peptide mass spectrometry.

Figure S14: Agnostic PTM profiling of itaconate-reacted purified BSA and KEAP1 in vitro, as studied by elastase peptide mass spectrometry.

Figure S15: Agnostic PTM profiling of itaconate-reacted purified BSA and KEAP1 in vitro, as studied by GluC peptide mass spectrometry.

Document S1: Pseudocode of the key spectral matching scoring functions in SAMPEI.

Table S1: List of chemically modified peptides agnostically identified using SAMPEI in the proteome of LPS stimulated RAW264.7 cells.

Table S2: List of peptides with putative itaconate adducts identified in RAW264.7 proteomes using variable modification search. 
Figure S1: SAMPEI parameterization using specific differentially alkylated proteomes.

a

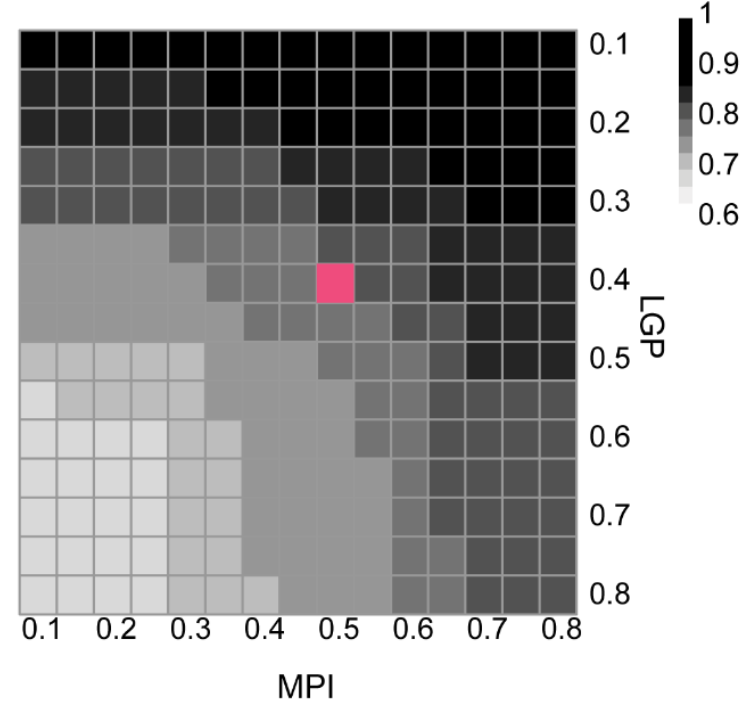

b

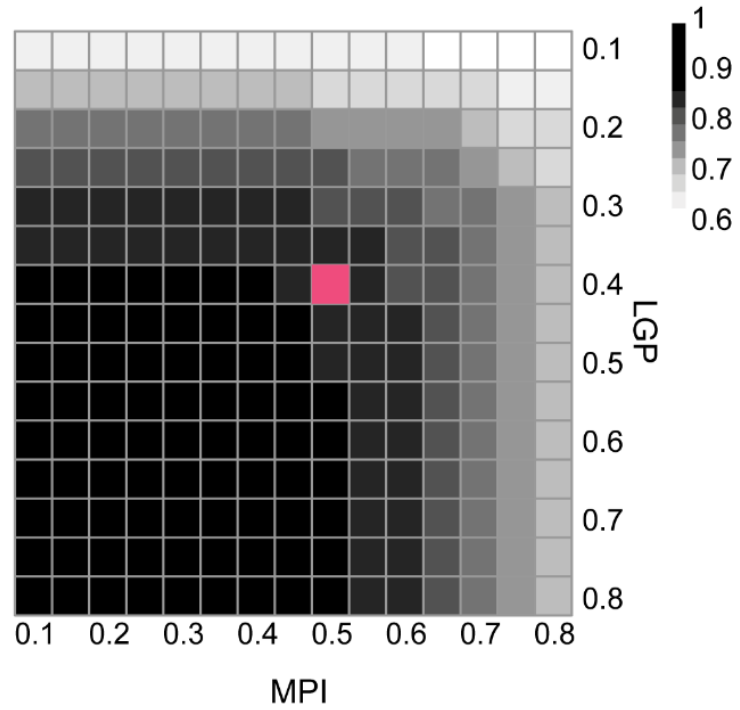

Empirical optimization of MPI and LGP parameters to maximize precision (a) and recall (b), setting propionylation as a fixed modification. Red symbols denote the default user-specified values. 
Figure S2: SAMPEI achieves sensitive and specific unbiased discovery of peptide modifications in complex cellular proteomes.
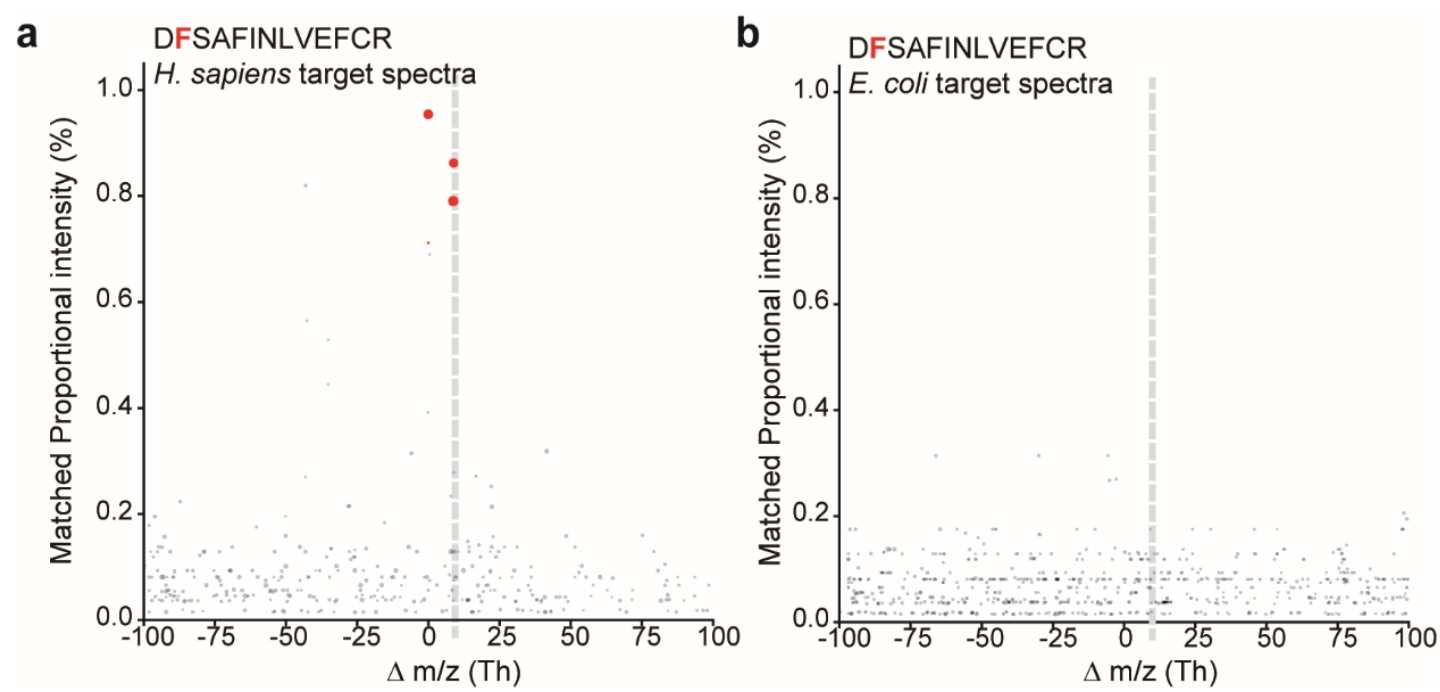

Fraction of matched spectra as a function of modification mass shift $(\mathrm{m} / \mathrm{z})$ demonstrating accurate identification of synthetically fluorinated D(fluoro-F)SAFINLVEFCR peptide (17.99 Da mass shift, corresponding to 8.995 Th for +2 charged ions, as marked by dotted gray line) using as queries human cell extract containing the unmodified peptide (a) or an E. coli proteome not containing the unmodified chemoform, and thus serves as a negative control (b). Red symbols denote query PSMs matched by SAMPEI to target spectra. M. Gray symbols denote matches of the fluorinated peptide with unrelated unmodified spectra. 
Figure S3: Absolute sensitivity of SAMPEI, ByOnic, MSFragger, and MaxQuant

a

Agnostic discovery of propionylated peptides

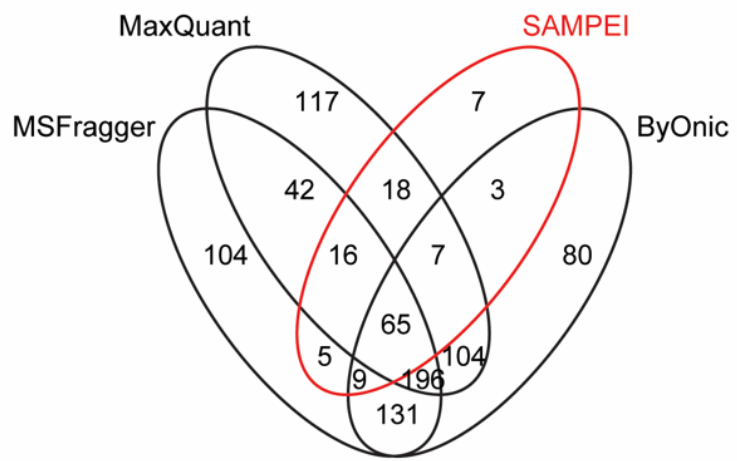

C

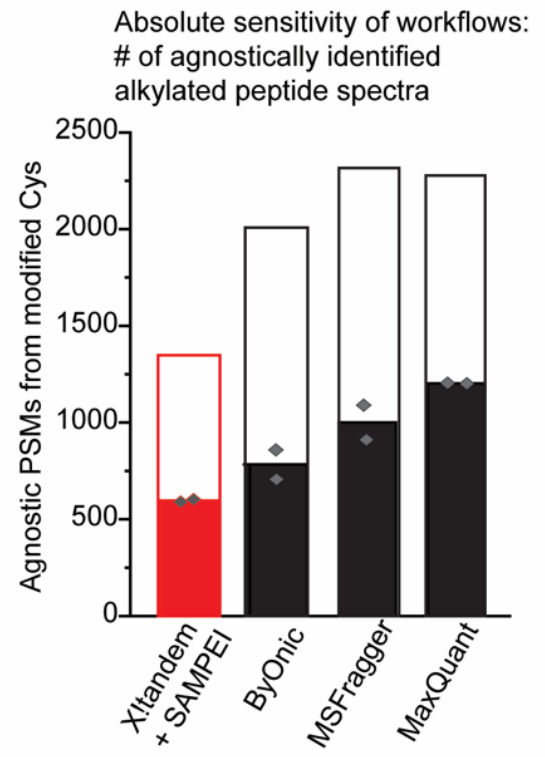

b

Agnostic discovery of carbamidomethylated peptides

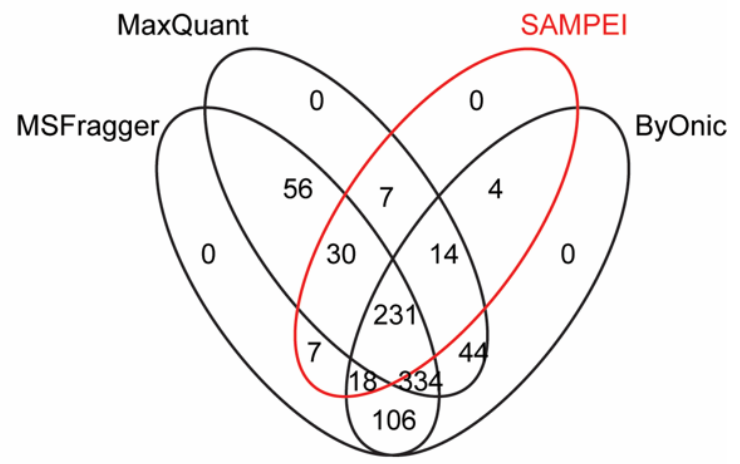

d

Sensitivity of agnostic PTM identification:

\# of alkylated peptide spectra identified by SAMPEI

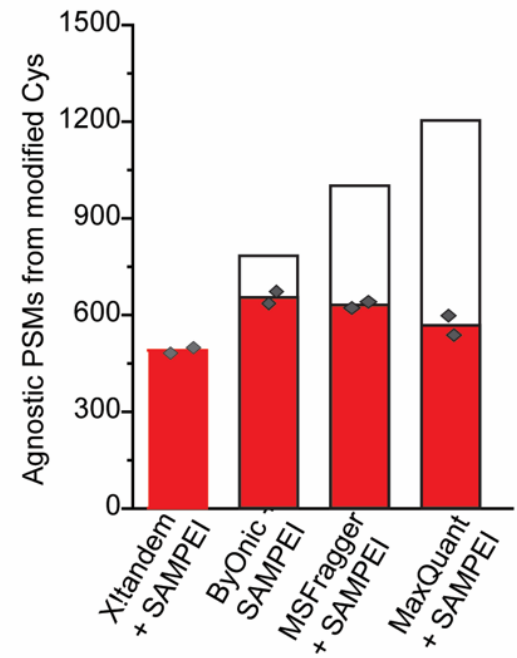

e
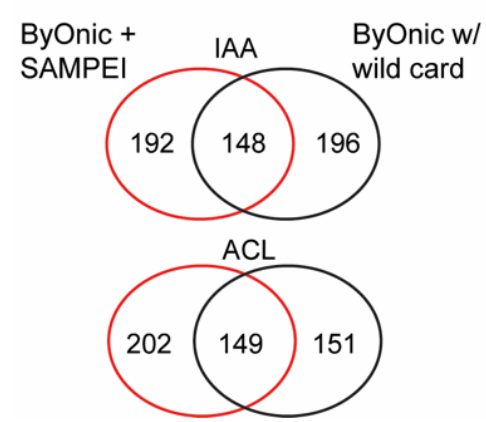

f

MSFragger + IAA MSFragger MaxQuant + IAA MaxQuant w/ SAMPEI IAA open search

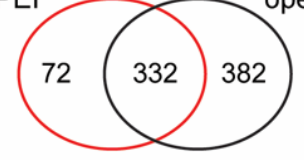
SAMPEI
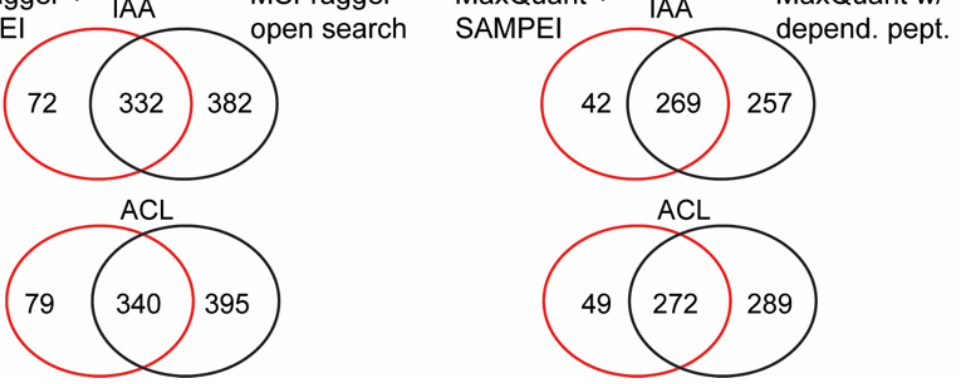

a,b. overlap between alkylated peptide sequences agnostically identified using X!Tandem plus SAMPEI, ByOnic with wild card, MSFragger open search, and MaxQuant with dependent peptides search. Venn diagrams show results relative to agnostic discovery of (a) propionylated 
peptides (from variable carbamidomethyl-cys searches) and (b) carbamidomethylated peptides (from variable proprionyl-cys searches). c. absolute sensitivity of software for agnostic discovery expressed as number of PSM with correctly modified cysteine residues (full bar). Empty bars denote the average $(\mathrm{n}=2)$ total number of cysteine-containing peptides detected using conventional searches. d. absolute sensitivity of SAMPEI discovery using as input PSM lists generated by X!tandem, ByOnic, MSFragger (closed search) and MaxQuant, respectively, expressed as number of PSM with correctly modified cysteine residues (full bar). Empty bars denote the average $(\mathrm{n}=2)$ number of PSM from modified peptides generated by the native functions for agnostic search. e-g. Overlap between the identified alkylated peptides discovered by SAMPEI and by the native functions for agnostic search of (e) ByOnic, (f) MSFragger, and (g) MaxQuant. 
Figure S4: Computational efficiency of SAMPEI.

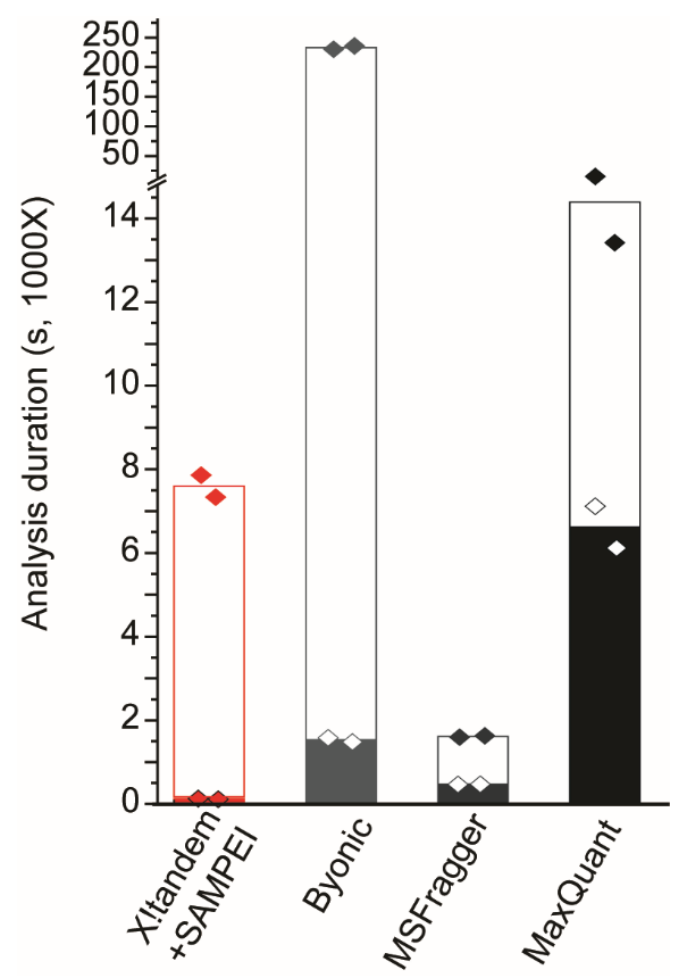

Duration of PTM tolerant analyses of 48,161 MS2 spectra by SAMPEI, MSFragger, Byonic, and MaxQuant. The duration of PTM restricted analysis is provided as full bar for each method.

Being necessarily sequential, the duration of SAMPEI and X!Tandem analyses were summed. 
Figure S5: Distribution of putative modified sites for agnostically identified PTMs.

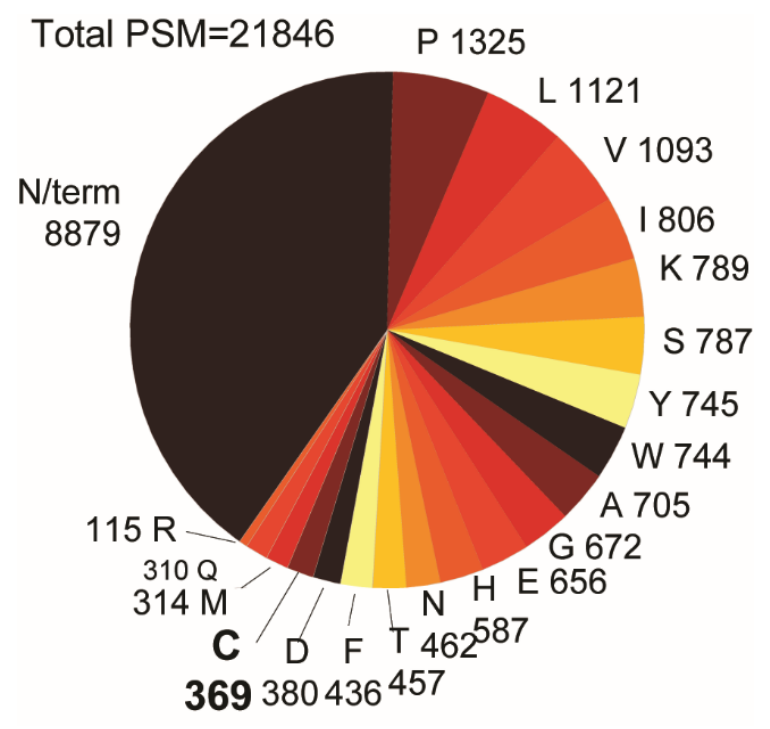

Numerical values denote number of modified peptide-spectral matches. 
Figure S6: Representative mass spectra pairs for peptides with either unmodified experimentally induced +57.02 Da modification or biologic +130.02 Da modification of peptide cysteines.

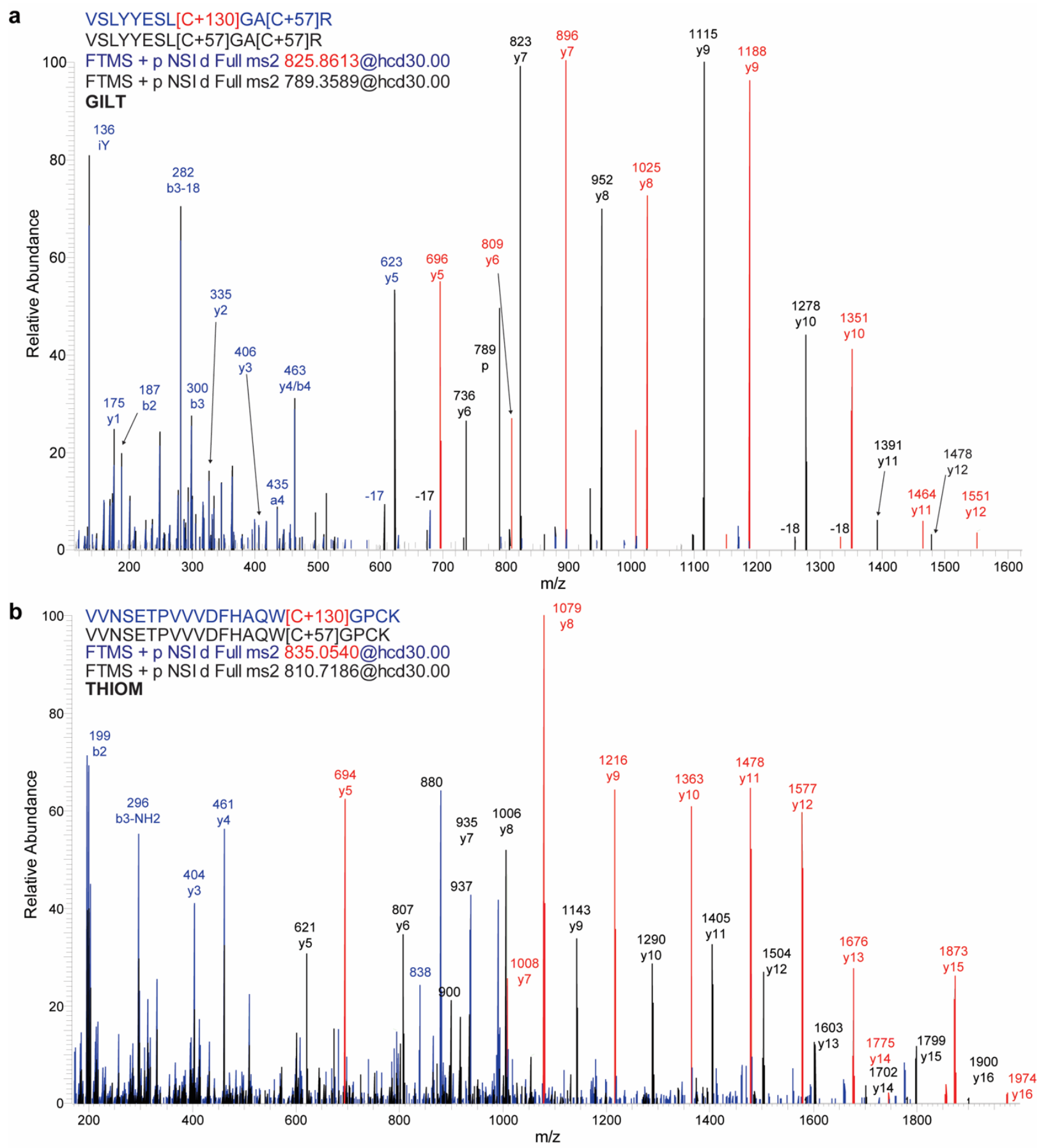

Representative mass spectra pairs of peptides with either unmodified cysteine (subject to experimental carbamidomethylation) or bearing +130.0266 Da modifications detected in GILT 
(a) and THIOM (b) proteins. High-resolution fragmentation mass spectra pairs were overlaid and annotated, showing diagnostic mass shift between modified peptides. Ions shared by both chemoforms are annotated in blue; ions solely matching the unmodified peptides are annotated in black; ions specific for the +130.0266 Da modified peptides are annotated in red. 
Figure S7: Representative mass spectra pairs for peptides with either unmodified experimentally induced +57.02 Da modification or biologic +146.02 Da modification of peptide cysteines.

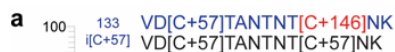

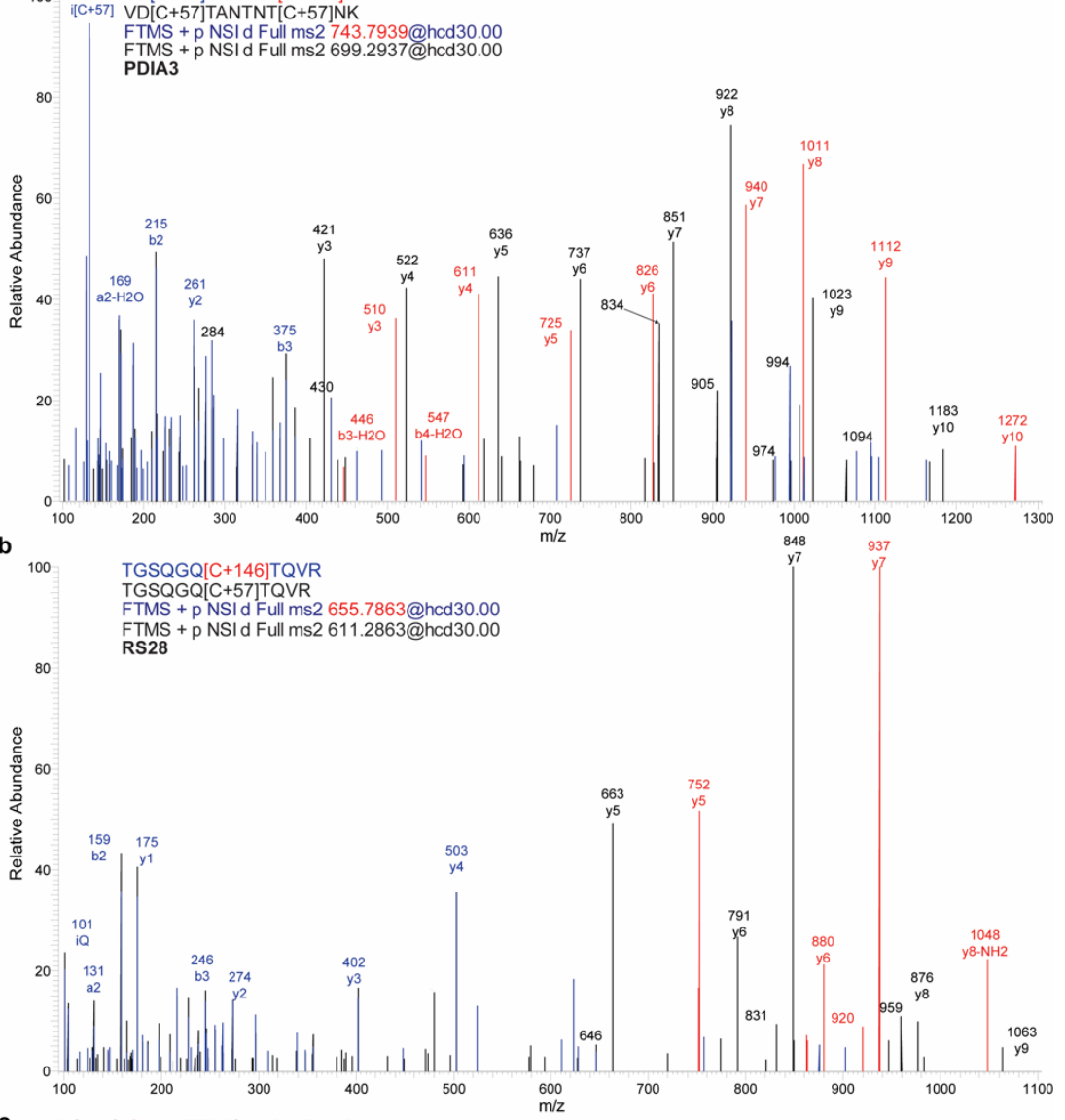

c IVSNAS[C+146]TTN[C+57]LAPLAK

IVSNAS[C+57]TTN[C+57]LAPLAK
FTMS + p NSI d Full ms2 954.9592@hcd30.00

FTMS + NS1d Full ms2 954.250@ @hcd30.00

${ }_{50}$ G3P $\quad 428.29$

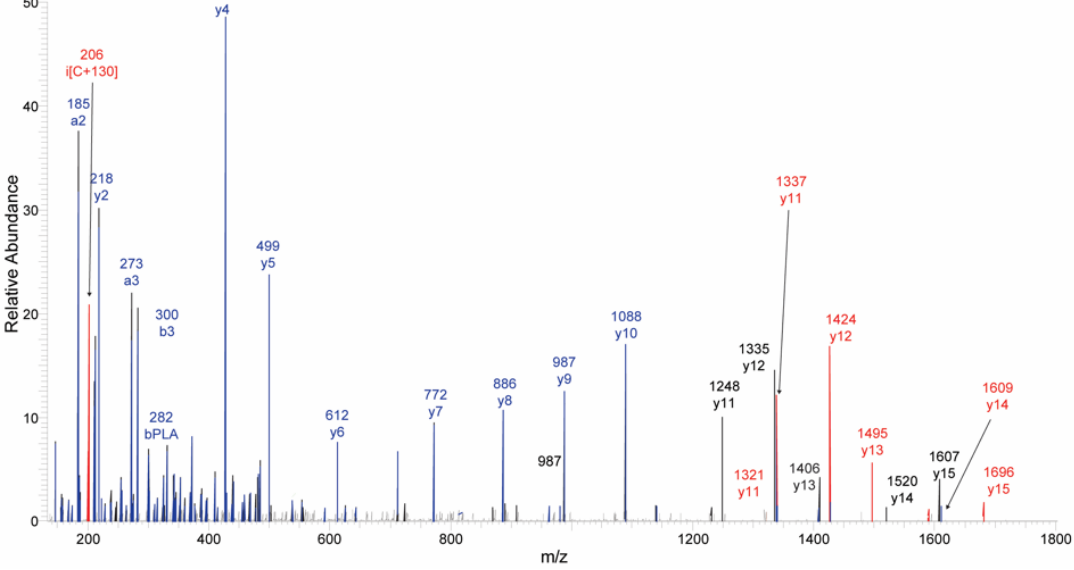


Representative mass spectra pairs of peptides with either unmodified cysteine (subject to experimental carbamidomethylation) or bearing +146.0215 Da modifications detected in PDIA3 (a), RS28 (b), and G3P (c) proteins. High-resolution fragmentation mass spectra pairs were overlaid and annotated, showing diagnostic mass shift between modified peptides. Ions shared by both chemoforms are annotated in blue; ions solely matching the unmodified peptides are annotated in black; ions specific for the +146.0215 Da modified peptides are annotated in red. 
Figure S8: Spectral quality of chemically modified peptides detected as multiple or single modification isoforms.

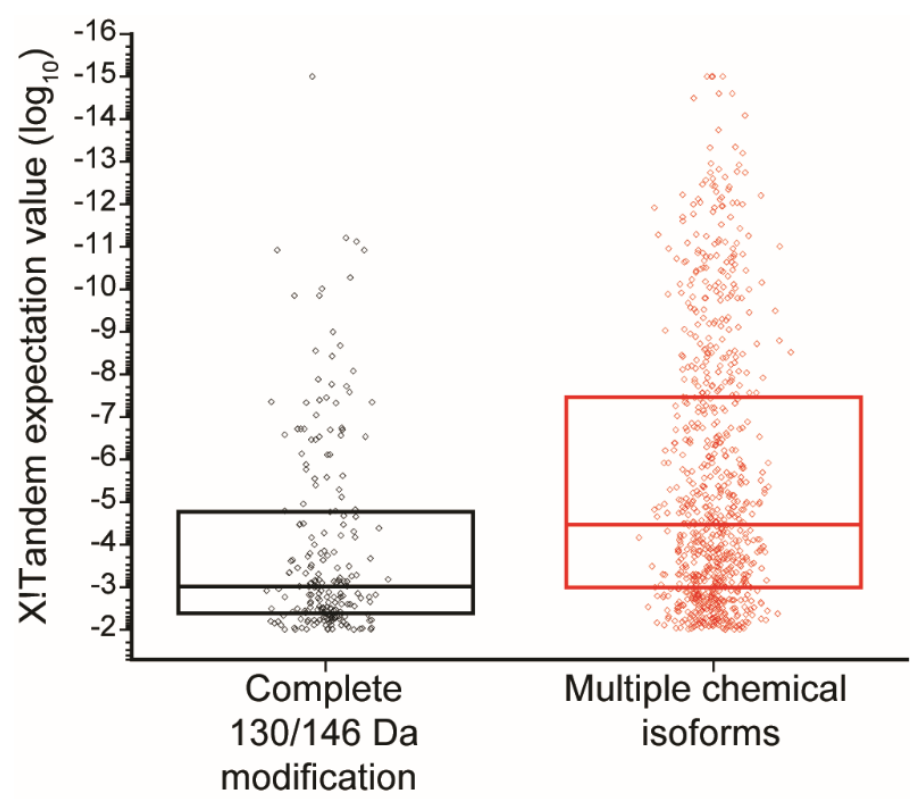

Statistical confidence of PSMs (expressed as X!Tandem expectation value) from modified peptides detected only with 130.03 or 146.02 Da adducts (black) versus peptides detected also in their unmodified form (red), and thus amenable for SAMPEI identification. Boxes represent median, $25^{\text {th }}$ and $75^{\text {th }}$ percentile. 
Figure S9: Quantification of itaconate production by LPS-stimulated macrophages.

a

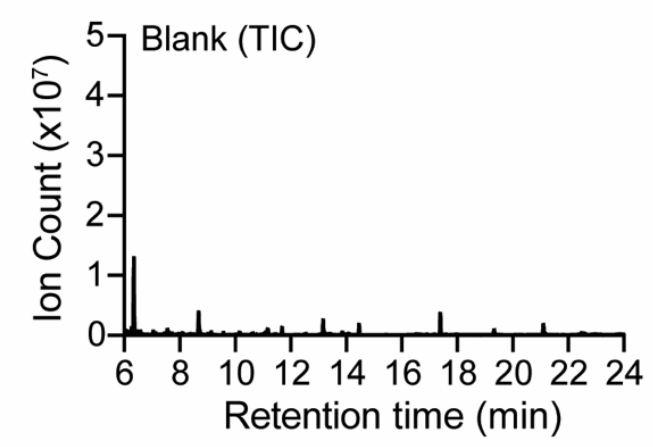

b

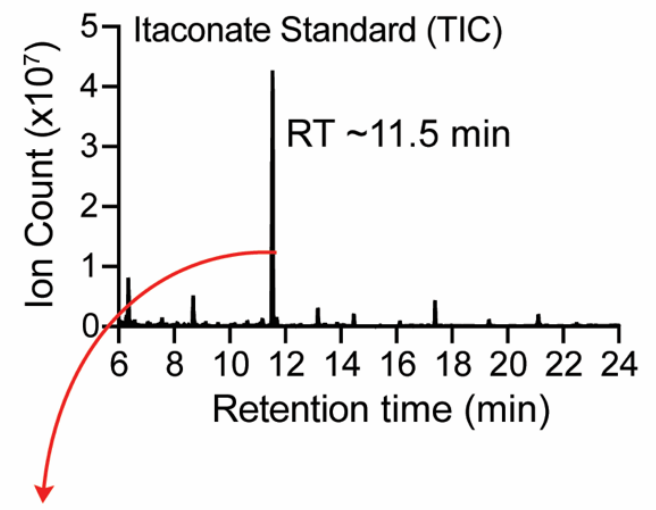

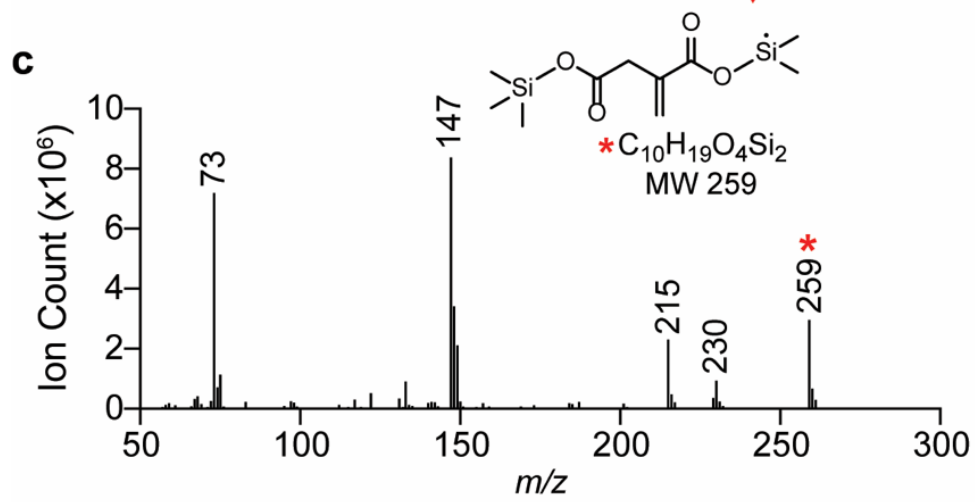

d<smiles>C=C(CC(=O)O)C(=O)O</smiles>
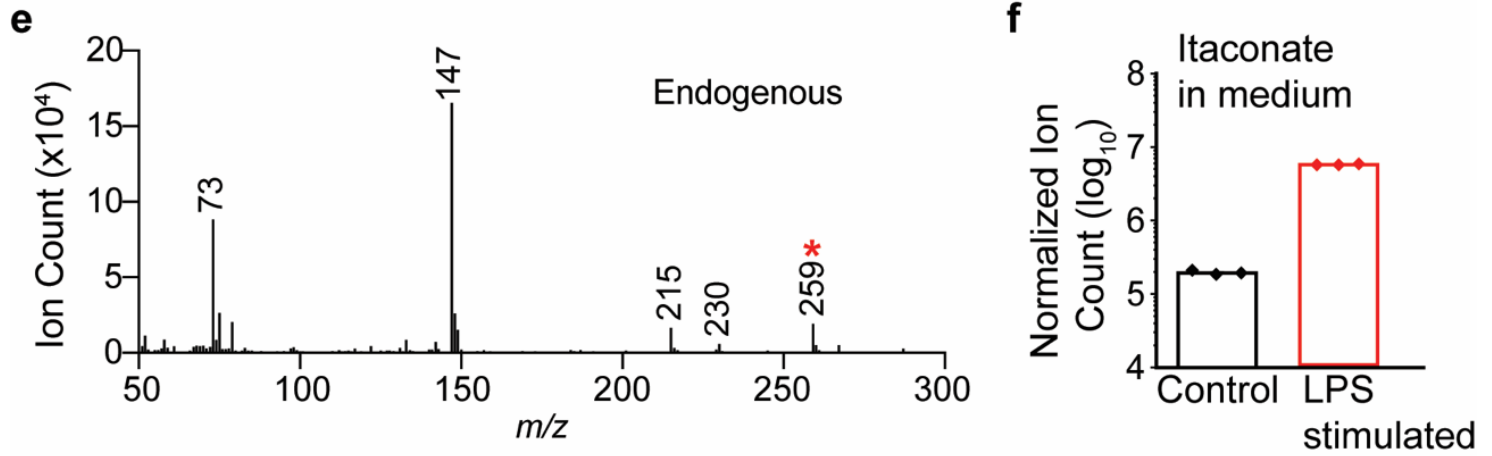

Total ion chromatograms (TIC) for blank solvent (a) or pure bis-(trimethylsilylated) itaconate standard (b) measured by GC-MS (see Methods for details). The specific peak for bis(trimethylsilyl)-itaconate was detected at a retention time of $\sim 11.5$ minutes. c,e. Mass spectrum for the bis-(trimethylsilyl)-itaconate (structure in inset) peak at retention time 11.5 minutes detected in LPS-stimulated RAW264.7 macrophages (c) and unstimulated stage (e); m/z 259 was used for subsequent itaconate quantification and $\mathrm{m} / \mathrm{z} 215$ as a confirmatory ion. $\mathrm{d}$. Structure of 
underivatized itaconate. f. Quantification of itaconate production by RAW264.7 macrophages upon LPS stimulation as measured by LC-MS in medium ( $\mathrm{n}=3$ biologic replicates). 
Figure S10: IRG1 immunoblot - entire membrane.
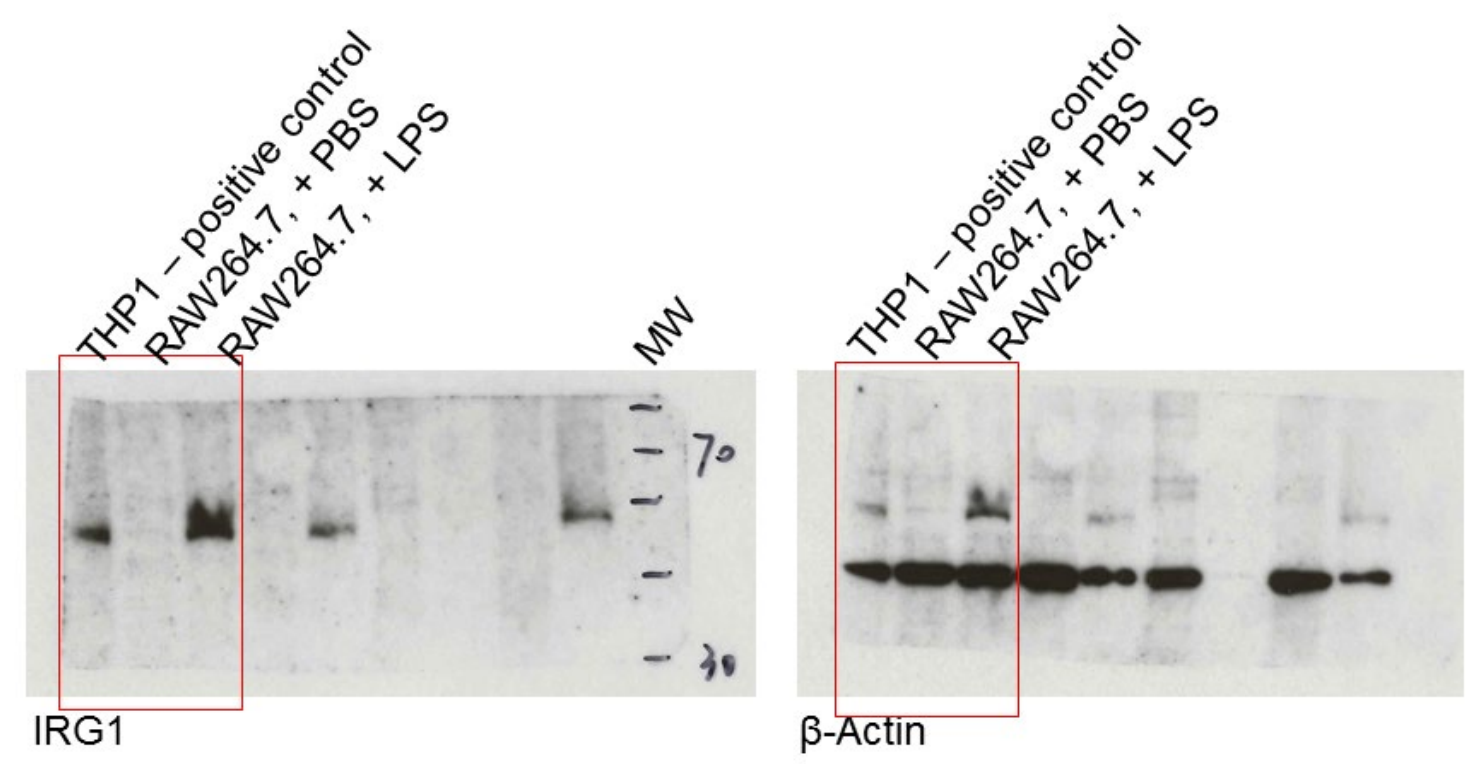

Entire membrane for the Western blot in figure 5c. The same membrane was probed first with anti-IRG1 antibody, and then with anti-actin antibody as loading control. Stimulated THP1 cells, which express IRG1, were used as positive control. Red box indicates the portion of the blot considered to confirm IRG1 induction in stimulated RAW264.7 cells. 
Figure S11: GO term enrichment of proteins with +130.02 Da and +146.02 Da peptide modifications.

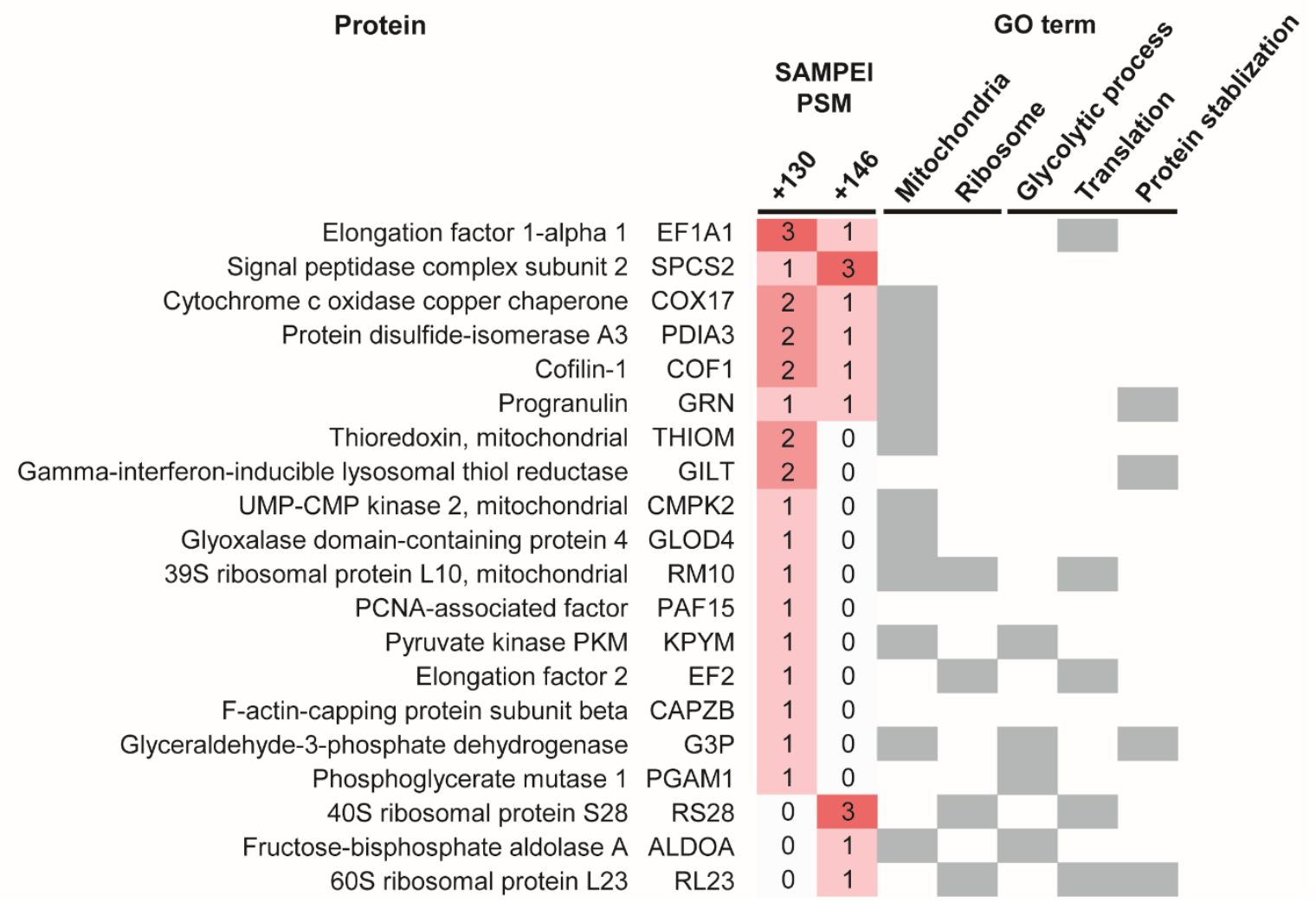


Figure S12: Schematic of itaconate reactions with purified BSA and KEAP1 proteins, followed by proteolysis and peptide chromatography and high-resolution tandem mass spectrometry.

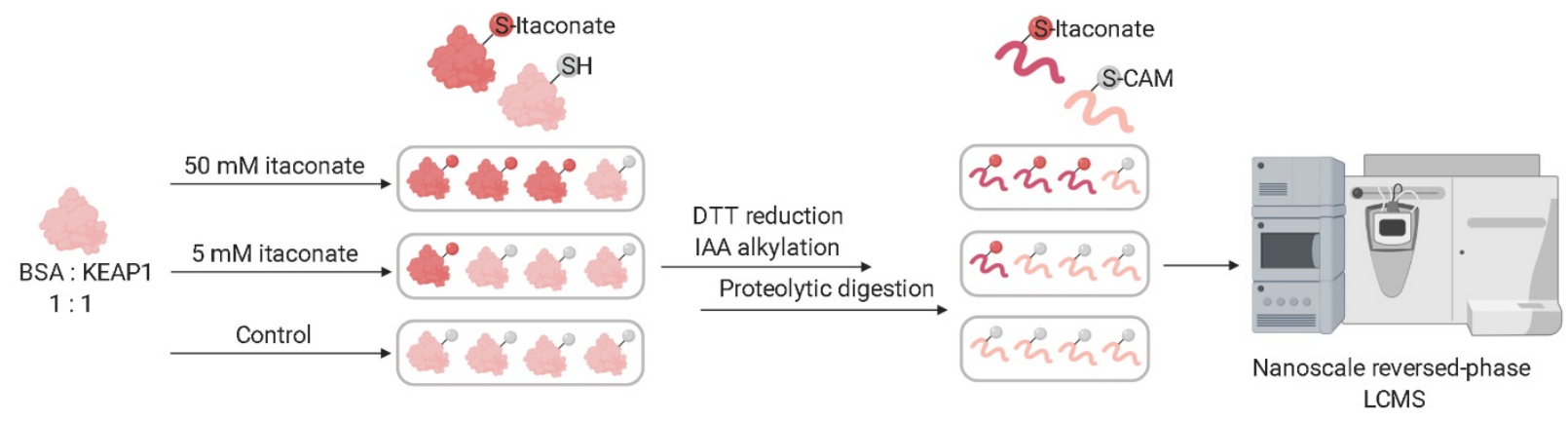


Figure S13: Agnostic PTM profiling of itaconate-reacted purified BSA and KEAP1 in vitro, as studied by tryptic peptide mass spectrometry.

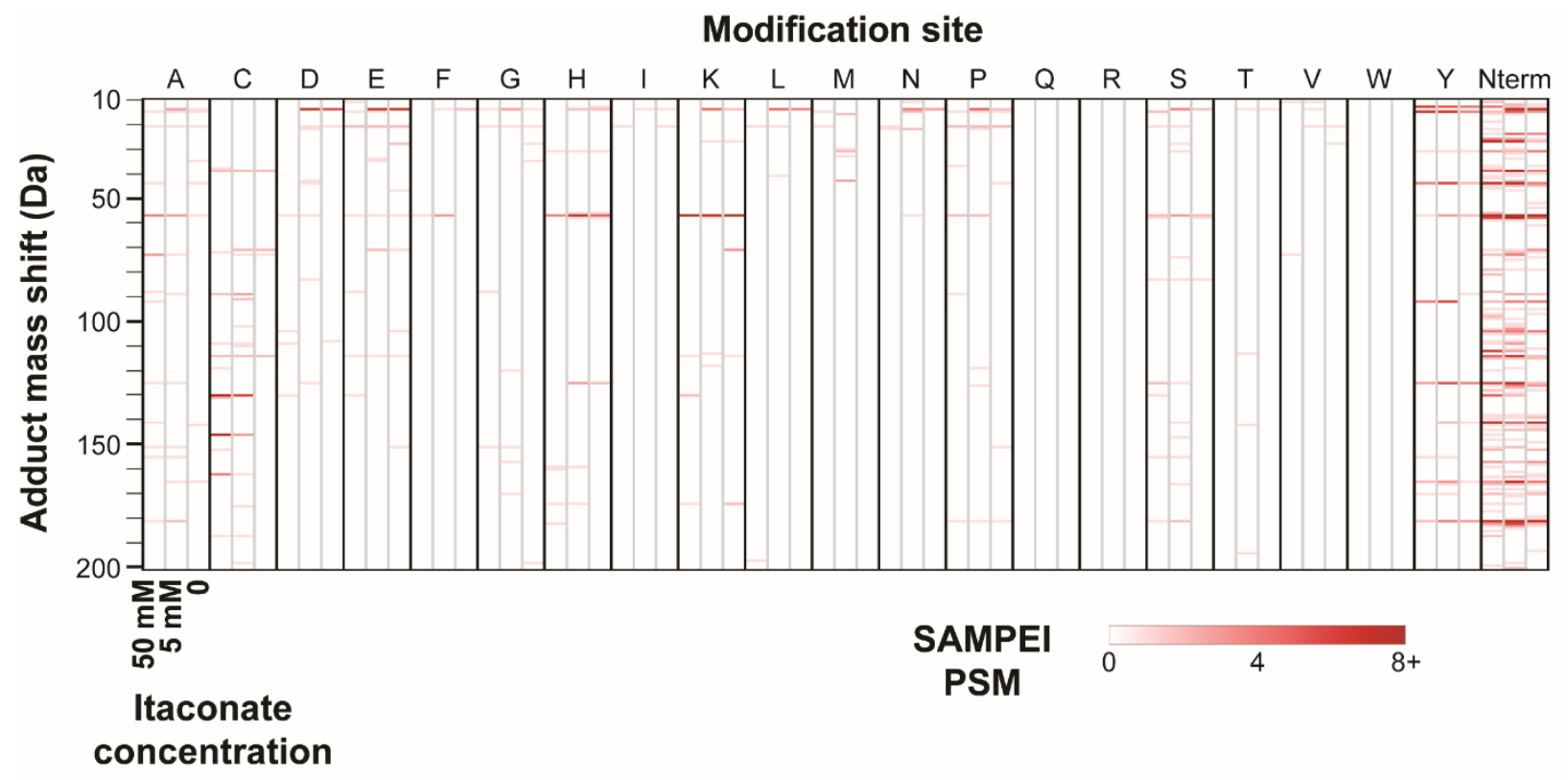

Frequency of PTM mass shifts of BSA and KEAP1 as a function of increasing concentrations of itaconic acid. For each putative localization, the three columns indicate adducts observed upon treatment with $50 \mathrm{mM}$ (left column), $5 \mathrm{mM}$ (middle) and $0 \mathrm{mM}$ control itaconic acid (right column). Intensity of red marking indicates prevalence, expressed as number of SAMPEI PSMs. 
Figure S14: Agnostic PTM profiling of itaconate-reacted purified BSA and KEAP1 in vitro, as studied by elastase peptide mass spectrometry.

a

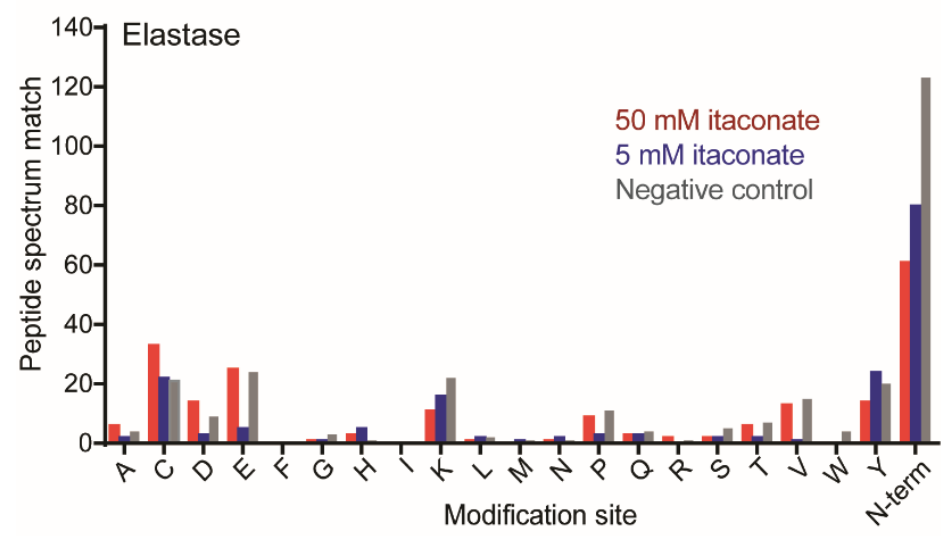

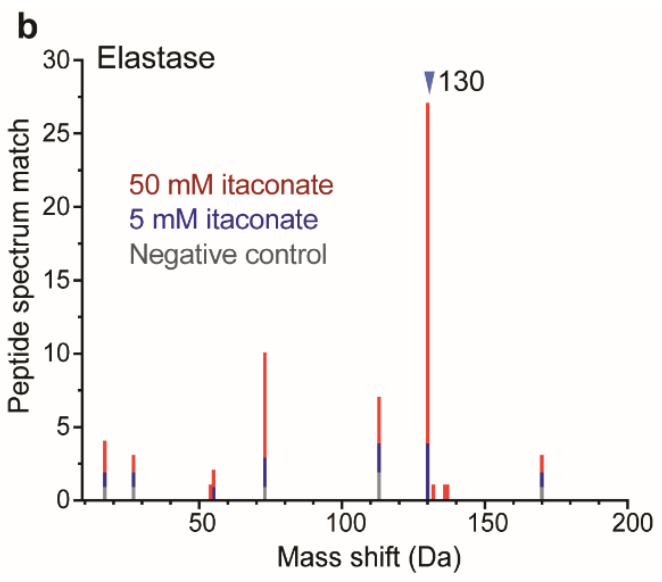

C

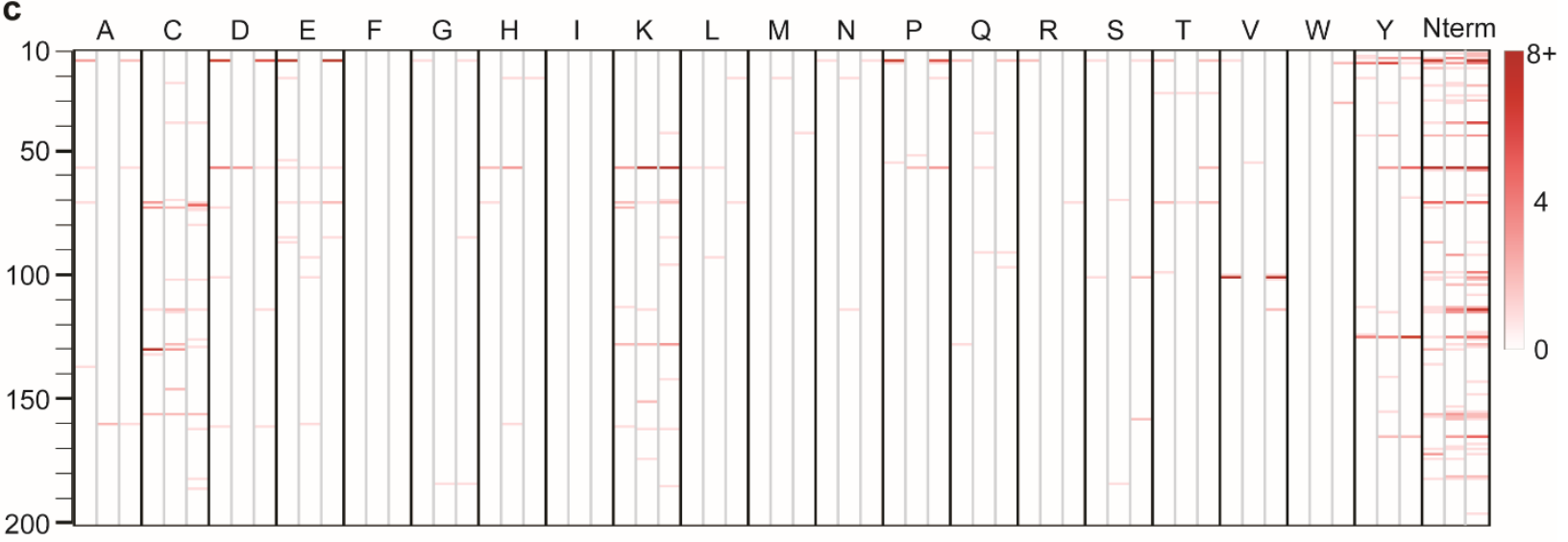

a. Localization of non-canonical modifications of BSA and KEAP1, showing dependency of cysteine modifications on itaconate concentration. b. Frequency of observed adducts on BSA and KEAP1 treated with itaconate in vitro and negative control (as stacked column), showing dosedependency of +130.02 Da adducts. c. Frequency of PTM mass shifts on BSA and KEAP1 amino acids treated with different doses of itaconic acid. For each localization, the three columns indicate adducts profiling obtained after treatment with $50 \mathrm{mM}$ itaconate (left column), $5 \mathrm{mM}$ itaconate (middle) and negative control (right column). Intensity of red marking indicates prevalence, expressed as number of SAMPEI PSMs. 
Figure S15: Agnostic PTM profiling of itaconate-reacted purified BSA and KEAP1 in vitro, as studied by GluC peptide mass spectrometry.

a

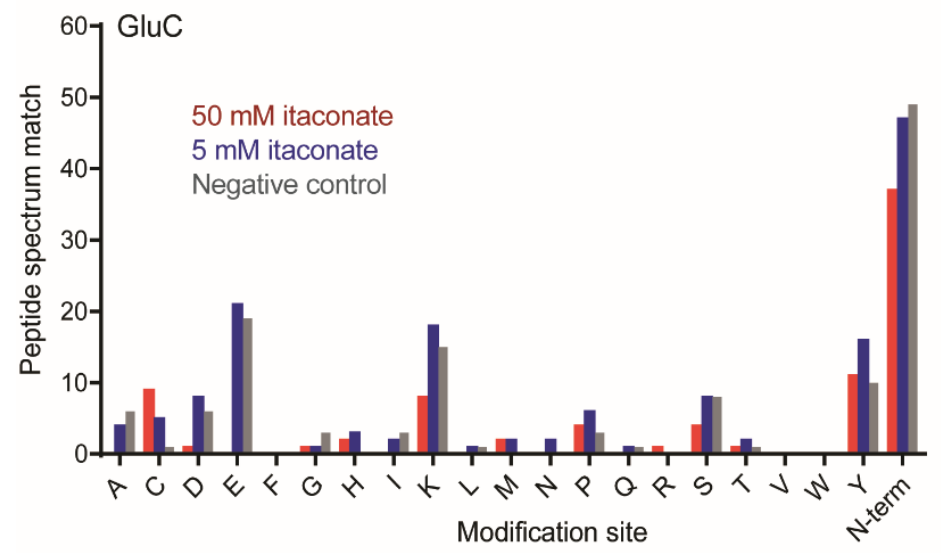

b

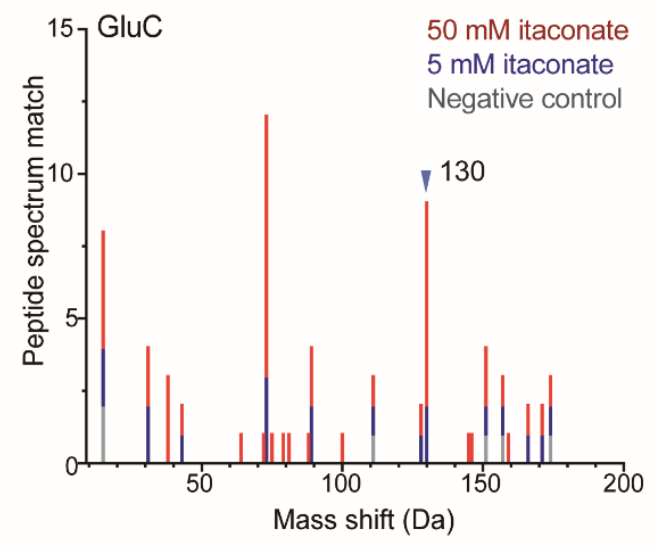

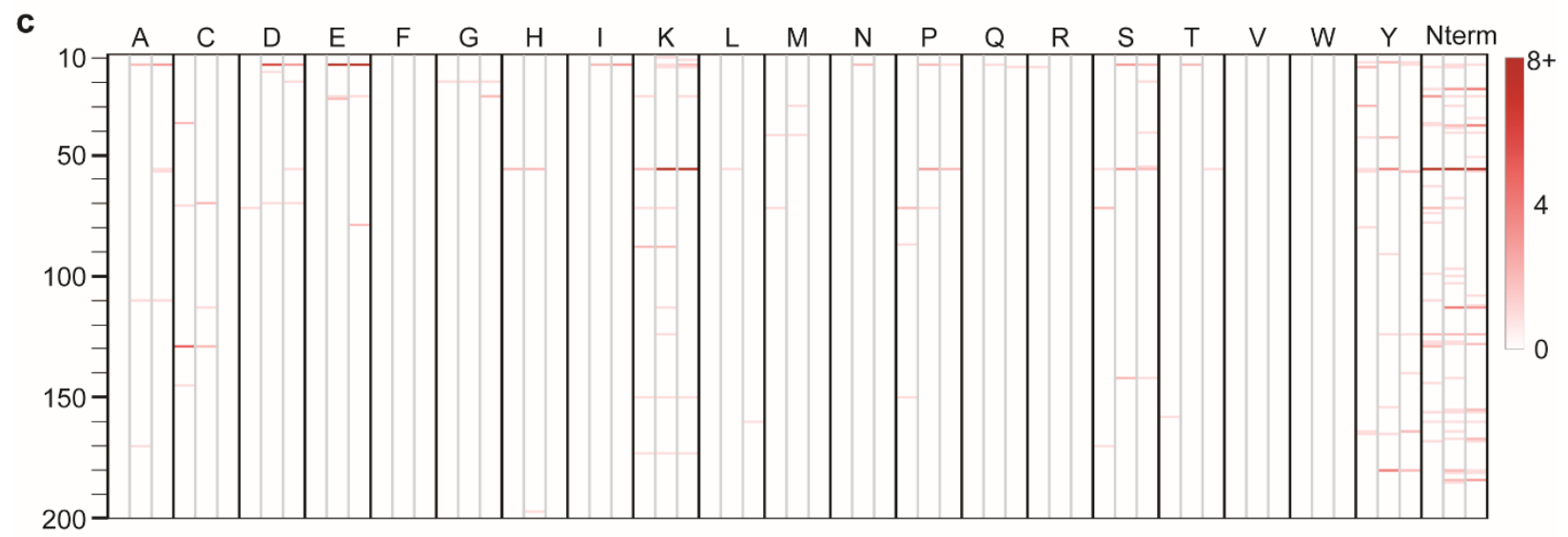

a. Localization of non-canonical modifications BSA and KEAP1, showing dependency of cysteine modifications on itaconate concentration. b. Frequency of observed adducts on BSA and KEAP1 treated with itaconate in vitro and negative control (as stacked column), showing dose-dependency of +130.02 Da adducts. c. Frequency of PTM mass shifts on BSA and KEAP1 amino acids treated with different doses of itaconic acid. For each localization, the three columns indicate adducts profiling obtained after treatment with $50 \mathrm{mM}$ itaconate (left column), $5 \mathrm{mM}$ itaconate (middle) and negative control (right column). Intensity of red marking indicates prevalence, expressed as number of SAMPEI PSMs. 
Document S1: Pseudocode of the key spectral matching scoring functions in SAMPEI.

Algorithm 1: Preliminary filtering of spectra

Inputs: Query and target vector ( $\mathrm{q}[\mathrm{:}]$ and $\mathrm{t}[\mathrm{:}]$ ) of $\mathrm{m} / \mathrm{z}$-abundance pairs

Error tolerance (error)

Precursor mass difference between query and target scans (diff)

Result: Matched query intensity (matched_query)

for each $\mathrm{m} / \mathrm{z}$-abundance pair in $\mathrm{t}[\mathrm{:}]$ :

total_target $+=$ target_abundance

for each $\mathrm{m} / \mathrm{z}$-abundance pair( $\left.\mathrm{v}_{-} \mathrm{q}\right)$ in $\mathrm{q}[\mathrm{:}]$ :

total_query+=query_abundance

for $i:=l$ to the length of $t[:]$ :

if absolute difference between $\mathrm{m} / \mathrm{z}$ of $\mathrm{v}_{-} \mathrm{q}$ and $\mathrm{m} / \mathrm{z}$ of $\mathrm{t}[\mathrm{i}]$ is less than error $/ \mathrm{le} 6 * \mathrm{~m} / \mathrm{z}$ of $\mathrm{v} \_\mathrm{q}$ or absolute difference between $\mathrm{m} / \mathrm{z}$ of $\mathrm{v} \_\mathrm{q}$ and $\mathrm{m} / \mathrm{z}$ of $\mathrm{t}[\mathrm{i}]$ + diff is less than error $/ l_{e} 6 * \mathrm{~m} / \mathrm{z}$ of $\mathrm{v}_{-} \mathrm{q}$ :

matched_query $+=$ abundance of $v_{-} q$

remove $t[i]$

break

if total_query $>0$ :

matched_query /= total_query

return matched_query 
Algorithm 2: Matched Proportional Intensity (MPI)

Inputs: Peptide sequence of query scan (pep)

Query scan (scan_str)

Modifications of query scan (query_mod)

Precursor mass difference between query and target (diff_dalton)

Charge of target scan (charge)

Error tolerance (fragment)

Error type (fragment_type)

Minimum fragment $\mathrm{m} / \mathrm{z}$ threshold (frag_mz_min)

Maximum number of peaks allowed (max_peaks_per_scan)

list of $\mathrm{m} / \mathrm{z}$ abundance pairs (mgf_table)

Monoisotopic masses (masses)

Result: Maximum intensity matching (matched_intensity_max)

Full modifications assigned (mod_with_maxmatchedintensity)

Unique modification by SAMPEI (mod_with_maxmatchedintensity_u)

if query_mod $\neq$ ":

for $\mathrm{j}:=1$ to the length of modifications in query_mod:

mod_pos_mass[aa_position[j]]=mass associated with aa_position mod_pos.append(aa_position[j])

for $i:=l$ to the length of pep:

mod_pos_mass_new $=$ mod_pos_mass

mod_pos_mass_unique $=\{\}$

if $\operatorname{str}(i+1)$ not in mod_pos:

mod_pos_mass_new[str(i+l)] $=$ str(diff_dalton)

mod_pos_mass_unique[str(i+l)]=str(diff_dalton) 
if $\operatorname{str}(i+1)$ in mod_pos:

mod_pos_mass_new[str(i+1)]=mod_pos_mass[str(i+1)]+diff_dalton mod_pos_mass_unique[str(i+1)]=mod_pos_mass[str(i+1)]+diff_dalton

$\bmod ="$

mod_new_only="

for $\mathrm{j}$ in mod_pos_mass_new: $\bmod =\bmod +\bmod \_$pos_mass_new $[j]$

for $\mathrm{u}$ in mod_pos_mass_unique:

mod_new_only=mod_new_only+mod_pos_mass_unique[u]

fragments $=[]$

fragments=calc_peptide_fragments(fragments, pep, mod,charge,'by', masses)

for $\mathrm{c}:=$ charge to 0 by -1 :

mass_ion_t $\mathrm{t}=$ precursor_mass $+\mathrm{c}^{*}$ masses['Proton'] $) /(1.0 * \mathrm{c})$

fragments.append(mass_ion_t)

for $1:=1$ to 3 :

mass_ion_t $\mathrm{t}=$ precursor_mass

$1 *$ masses['H2O']+c*masses['Proton'] $) /\left(1.0{ }^{*} \mathrm{c}\right)$

fragments.append(mass_ion_t)

mass_ion_t $\mathrm{t}=$ precursor_mass

$1 *$ masses['NH3'] $+\mathrm{C} *$ masses['Proton'] $) /(1.0 * \mathrm{c})$

fragments.append(mass_ion_t)

matched_intensity=select_fragments_scan(scan_str,frag_mz_min,mgf_pepmatch_ion s,fragments, max_peaks_per_scan, mgf_table)

if matched_intensity is greater than matched_intensity_max: 
matched_intensity_max=matched_intensity

mod_with_maxmatchedintensity $=\bmod$

mod_with_maxmatchedintensity_u=mod_new_only

return matched_intensity_max;

mod_with_maxmatchedintensity;

mod_with_maxmatchedintensity_u 
Algorithm 3: Largest Gap Percentage (LGP)

Inputs: Peptide sequence(pep)

b-ions supporting the peptide assignment (sequence_evidence_b)

$\mathrm{y}$-ions supporting the peptide assignment (sequence_evidence_y)

Result: Longest discontinuity in the ion series (largest_gap)

for $k:=1$ to the length of (pep)-1:

if sequence_evidence_b[k] less than 0 or sequence_evidence_y[length of (pep)-2-k]>0:

$$
\text { gap }=0
$$

else:

$$
\text { gap }+=1
$$

if largest_gap<gap:

largest_gap=gap

return largest_gap 